\title{
Isolation and Molecular Characterization of Abiotic Stress Tolerant Plant Growth Promoting Pseudomonas spp. from Different Rhizospheric Soils of Telangana State, India
}

\author{
K. Damodarachari ${ }^{1 *}$, R. Subhash Reddy ${ }^{1}$, S. Triveni ${ }^{1}$, CH. V. Durga Rani ${ }^{2}$ and M. Sreedhar ${ }^{3}$
}

${ }^{1}$ Dept. of Agricultural Microbiology \& Bioenergy, College of Agriculture, ${ }^{2}$ Dept. of Molecular Biology \& Biotechnology, Institute of Biotechnology, ${ }^{3}$ Quality Control Laboratory, Professor JayashankarTelangana State Agricultural University, Rajendra nagar, Hyderabad, Telengana State (500 030), India

\section{Corresponding Author}

K. Damodarachari

e-mail: damuagmicro2012@gmail.com

\author{
Article History \\ Article ID: AR1913 \\ Received in $06^{\text {th }}$ October, 2018 \\ Received in revised form $22^{\text {nd }}$ October, 2018 \\ Accepted in final form $23^{\text {rd }}$ October, 2018
}

\begin{abstract}
The present investigation was carried out to isolate rhizobacteria from different rhizospheric soils of Telangana state, India and screened for plant growth promoting properties such as mineral nutrient solubilization ( $P, K, Z n)$, IAA production, ACC deaminase, EPS production, biocontrol activity and tolerance to different abiotic stresses such as $\mathrm{pH}$, temperature, salt, drought and heavy metals in vitro conditions. Based on cultural, morphological and biochemical characterization, it was found that forty four were of Pseudomonas spp. Among the forty four (44) Pseudomonas isolates, twenty eight (28) isolates were showing plant growth promoting properties. The results of different in vitro abiotic stress tolerance of Pseudomonas isolates, four isolates were showed growth at pH range from 4-10 (PS 1, PS 4, PS 26, PS 40). Five isolates were showed tolerance to 1.5 to $20 \%$ of $\mathrm{NaCl}$ concentration (PS 4, PS 15, eight isolates were showed tolerance from 1.5 to $15 \%$ of $\mathrm{NaCl}$ concentration (PS 1, PS 11, PS 18, PS 26, PS 30, PS 31, PS 32, PS 40), three isolates showed tolerance to temperature from $20^{\circ} \mathrm{C}-50{ }^{\circ} \mathrm{C}$ (PS 11, BS 15, BS 40), nine isolates were showed tolerance to temperature from $20^{\circ} \mathrm{C}-45^{\circ} \mathrm{C}$ (PS 1, PS $4, \mathrm{PS} 21, \mathrm{PS} 23, \mathrm{PS} 28, \mathrm{PS} 30, \mathrm{PS} 32, \mathrm{PS}$ $34, \mathrm{PS} 37)$, three isolates showed tolerance to water potential from $-0.05 \mathrm{Mpa}$ to $-0.73 \mathrm{Mpa}$ (PS 4, PS 15, BS 34 ).PS 15 was found efficient in $\mathrm{Zn}$ - solubilization with considerable other PGP properties. It was tolerant to $\mathrm{pH}$ stress $(4,6)$, temperature stress, salt stress, drought stress and heavy metals As and Cd only. PS 1 showed good P-solubilization, K-solubilization, Zn solubilization with biocontrol activity. It tolerated $\mathrm{pH}$ stress, temperature stress, salt stress, drought stress and tolerant to heavy metal toxicity. Performing environmental parameters for bacterial growth is also showing that bacteria can easy to survive in different environmental condition.
\end{abstract}

Keywords: Pseudomonas, PGPR, pH, temperature, drought, heavy metal tolerance

\section{Introduction}

Increasing crop productivity and enhancing resistance or tolerance against various stress factors has become major aim for modern agriculture (Farooq et al., 2009). In sustainable agriculture, integrated pest management is considered the most efficient strategy to manage stress causing agents, such strategy rely on combining several approaches including using resistant varieties, crop rotation, monitoring pests, biocontrol and in severe situations employing pesticides in an attempt to keep stress agents under control (Wegulo, 2012). Biological control forms an integral part of the IPM strategy (Landaet al., 2004).

Root zone bacteria that have been found to have beneficial effects on various plants include species of the genera Arthrobacter, Azotobacter, Azospirillum, Bacillus, Enterobacter, Pseudomonas and Serratia (Gray and Smith, 2005), as well as Streptomyces spp. (Tokala et al., 2002; Dimkpa et al., 2008).
Although the exact mechanisms of plant growth stimulation remain largely speculative, it is known that they differ between bacterial strains and most certainly depend on the various compounds released by the different microorganisms. The literatureis replete with reports describing the production of the main phytohormone classes-auxins, cytokinins, gibberellins, abscisic acid and ethylene by plant growth promoting rhizobacteria (PGPR). The subject of PGPR elicited tolerance to abiotic stresses has been reviewed (Venkateswarlu et al., 2008). It has been shown that certain PGPR enhance plant stress tolerance through 1-aminocyclopropane-1carboxylate deaminase and provide significant protection to a wide range of plant species from the damage caused by various abiotic stress conditions. ACC breakdown and ethylene synthesis inhibition by ACCdeaminase decreases the damage of various stress situations by enhancing homeostasis in and around the plant root, especially at early stages of stress exposure (Ali et al., 2009). 
Increased incidence of abiotic and biotic stresses has become major cause for stagnation of productivity in principal crops. Besides high temperature, droughts, elevated $\mathrm{CO}_{2}$, extreme rainfall events, more floods, cold waves, heat waves, and cyclones are the other important natural disasters that cause serious economic losses, are likely to be witnessed as a result of global warming. These factor sare likely to cause serious negative impact on crop growth and yields and impose severe pressure on our land and water resources (Grover et al., 2011). Exopolysaccharides producing plant growth- promoting rhizobacteria can also bind cations including $\mathrm{Na}^{+}$. Therefore, an increase in the population density of EPS producing bacteria in the root zone is expected to decrease the content of $\mathrm{Na}^{+}$available for plant uptake, and thereby alleviate salt stress in plants growing in saline environments (Alamiet al., 2000). The present investigation is on the isolation of abiotic stress tolerant plant growth promoting rhizobacterial isolates followed by in vitro screening for tolerance to high $\mathrm{pH}$, temperature, salt conditions, osmotic stress, metal toxicity etc. and molecular identification of few efficient isolates. Such PGPR will be helpful for efficient of management of abiotic stress in crop production.

High saline soils deprive plants of water and sodic soils affect nutrient availability. No reports are available on the diversity of Pseudomonas spp. occurring in such stressed soils of India. Presence of fluorescent pseudomonads is ubiquitous and some reports are available on their occurrence in stressed environment like saline, sodic and semi arid soils (Djedidi et al., 2011; Egamberdieva, 2011). Pseudomonas fluorescens can produce a wide range of enzymes and metabolites that help plants withstand varied biotic and abiotic stresses (Saravanakumar et al., 2011). In India, commercial preparations of $P$. fluorescens is widely used for disease management in pulses, rice and vegetables. Presently $P$. fluorescens, P. putida. P. chloraphis, $P$. aureofacians and other species are widely used in agriculture as they play a crucial role in soil health and plant development (Weller et al., 2007).

\section{Materials and Methods}

\subsection{Soil samples, Bacterial isolation, and culture media}

The present experiment was carried out during 2016-17 at department of Agricultural microbiology and bioenergy, college of Agriculture, PJTSAU, Rhizospheric soils were collected from different places of Telangana such as normal soils, salt affected, drought soils. The details of soil samples were presented in Table 1. Forty four strains of pseudomonas were isolated from these soils. The strains were coded as PS1 to PS-44.For isolation of rhizobacteria, the method

\begin{tabular}{|c|c|c|c|c|c|c|c|c|}
\hline \multirow[t]{2}{*}{ Sampling site } & & \multirow[t]{2}{*}{$\mathrm{pH}$} & \multirow{2}{*}{$\begin{array}{c}\text { Electrical } \\
\text { conductivity } \\
\left(\mathrm{dS} \mathrm{m} \mathrm{m}^{-1}\right)\end{array}$} & \multirow{2}{*}{$\begin{array}{c}\text { Organic } \\
\text { carbon (\%) }\end{array}$} & \multicolumn{4}{|c|}{ Heavy metal concentrations ( $\mu \mathrm{g} \mathrm{ml}^{-1}$ ) } \\
\hline & & & & & Arsenic & Cadmium & Mercury & Manganese \\
\hline \multirow[t]{4}{*}{ Rangareddy } & Koheda & 8.1 & 0.30 & 0.40 & 3.08 & 3.80 & 17.00 & 11.20 \\
\hline & Ibrahimpatnm & 8.0 & 0.41 & 0.42 & 3.11 & 2.80 & 12.00 & 9.20 \\
\hline & Choutuppal & 7.9 & 0.50 & 0.43 & 3.00 & 2.00 & 17.00 & 12.20 \\
\hline & College farm, PJTSAU & 7.9 & 0.35 & 0.39 & - & - & - & 3.21 \\
\hline \multirow[t]{2}{*}{ Mahabubnagar } & Kalvakurthy & 7.8 & 0.40 & 0.41 & - & - & - & - \\
\hline & Bijenpaally & 7.2 & 0.24 & 0.36 & 3.08 & 3.80 & - & - \\
\hline \multirow[t]{2}{*}{ Wanaparthi } & Wanaparthi & 7.5 & 0.28 & 0.42 & - & - & - & \\
\hline & Pebbair & 8.0 & 0.45 & 0.37 & - & - & - & - \\
\hline Nagarkurnool & Kollapur & 7.4 & 0.24 & 0.50 & - & 2.00 & - & - \\
\hline \multirow[t]{2}{*}{ Shamshabad } & Maheswaram & 7.6 & 0.30 & 0.39 & 3.08 & 3.80 & 12.00 & 12.20 \\
\hline & Kandhukur & 7.5 & 0.26 & 0.38 & 3.08 & 3.80 & 8.00 & - \\
\hline \multirow[t]{2}{*}{ Yadagirigutta } & Bhongir & 7.8 & 0.30 & 0.43 & - & 8.00 & - & - \\
\hline & Yadagirigutta & 8.0 & 0.34 & 0.40 & - & - & 3.80 & - \\
\hline
\end{tabular}

proposed by Vlassak et al. (1992) was followed. The sample was agitated for 15 minutes on a vortex and serial dilutions of soil suspensions were prepared. $0.1 \mathrm{ml}$ of respective dilutions was spread on sterilized Petri plates containing specific media i.e.nutrient agar. The bacterial isolates were identified on the basis of morphological, physiological and biochemical characteristics according to the standard methods described in Bergey's manual of systematic bacteriology (Holt and Kreig, 1984).

2.2. Determination of mineral solubilization, IAA production, ACC Deaminase activity, EPS production, Siderophore production and antagonistic activity

Phosphate solubilization activity was determined using Pikovskaya's agar medium containing 0.5\% (W/V) $\mathrm{Ca}_{3}\left(\mathrm{PO}_{4}\right)_{2}$ 
(Pikovskaya, 1948), Potassium solubilization determined using Aleksandrov medium containing $0.2 \%$ potassium aluminum silicate (Prajapati and Modi, 2012), Zinc solubilization determined using Tris mineral salt medium containing $0.1 \%$ ZnO (Saravanan et al., 2003). IAA production (Duby and Maheswari, 2012), EPS production at stress induced conditions were checked (Ali et al., 2013), bacterial utilization of ACC as sole nitrogen source was screened using qualitative assay(Jacobson et al., 1994). Siderophore production was determined by the Chrome Azurol S plate assay (Schwyn and Neilands, 1987), antagonistic activity was verified by following dual culture technique (Skidmore and Dickinson, 1976)

\subsection{Screening for abiotic stress tolerance}

\subsubsection{Influence of $\mathrm{pH}$}

The $\mathrm{pH}$ of the culture medium was adjusted to 4, 6, 7, 8, 10 and 12 using sterile using buffers. $5 \mathrm{ml}$ TSB (Trypticase soya broth) culture medium having different $\mathrm{pH}$, and $0.1 \mathrm{ml}$ bacterial suspension $\left(10^{8}-10^{9}\right.$ cells $\left.\mathrm{ml}^{-1}\right)$ was poured in sterile culture tubes in three replicates for each $\mathrm{pH}$ and incubated in shaker incubator at $120 \mathrm{rm}^{-1}$ was measured at $600 \mathrm{~nm}$.

\subsubsection{Influence of salt concentration}

Screening for salinity tolerance of isolated bacterial isolates by the inducing of different $\%$ of $\mathrm{NaCl}$ concentrations from $1.5 \%$, $5 \%, 10 \%, 15 \%$ and $20 \%$ were checked.

\subsubsection{Influence of temperatures}

$0.1 \mathrm{ml}$ of bacterial suspension $\left(10^{8}-10^{9}\right.$ cells $\left.\mathrm{ml}^{-1}\right)$ was poured into the vials containing $5 \mathrm{ml} \mathrm{TSB}$ culture medium and culture in incubators at $20^{\circ} \mathrm{C}, 30^{\circ} \mathrm{C}, 40^{\circ} \mathrm{C}, 45^{\circ} \mathrm{C}$ and $50{ }^{\circ} \mathrm{C}$ in three replicates for each. After $24 \mathrm{hrs}$ of culture, their absorbance was measured at $600 \mathrm{~nm}$.

\subsubsection{Influence of drought}

Trypticase soya broth (TSB) with different water potentials $(-0.05,-0.15,-0.30,-0.49$, and $-0.73 \mathrm{MPa})$ was prepared by adding appropriate concentrations of polyethylene glycol (PEG 6000) (Michel and Kaufmann 1973; Sandhya et al., 2009) and was inoculated with $1 \%$ of overnight raised bacterial cultures in TSB. Osmotic potential of broth media was measured by osmometer Three replicates of each isolate with each concentration were prepared. After incubation at 28 ${ }^{\circ} \mathrm{C}$ under shaking conditions (120 rpm) for $24 \mathrm{~h}$, growth was estimated by measuring the optical density at $600 \mathrm{~nm}$ using a spectrophotometer.

\subsubsection{Influence of heavy metals}

Freshly prepared agar plates were amended with various soluble heavy metal salts namely $\mathrm{As}, \mathrm{Cd}, \mathrm{Hg}$ and $\mathrm{Mn}$ at concentration of 50 and $100 \mathrm{~g} \mathrm{~m} \mathrm{~m}^{-1}$ were inoculated with overnight grown cultures. Heavy metal tolerance was determined by appearance of bacterial growth after incubating the plates at room temperature for 24-48 hours.

\subsection{Molecular identification of efficient stress tolerance plant growth promoting bacterial isolates}

The bacterial strains were identified using standard method of $16 \mathrm{~S}$ rRNA gene sequencing. DNA template was prepared by picking individual colony of each strain and amplification of $16 \mathrm{~S}$ rRNA gene was carried out by PCR. PCR amplification of DNA was done using universal primers: 27 F AGAGTTTGATCCTGGCTCAG and $1492 \mathrm{R}$ :TACGGTTACCTTGTTACGACTT. Reaction mixture (25 $\mu \mathrm{L})$, prepared for full-length 16S rRNA geneamplification was initially denatured at $94^{\circ} \mathrm{C}$ for $2 \mathrm{~min}$, followed by 30 cycles consisting of denaturation at $94^{\circ} \mathrm{C}$ for $1 \mathrm{~min}$; primer annealing at $52{ }^{\circ} \mathrm{C}$ for $1: 30 \mathrm{~min}$. and primerextension at $72{ }^{\circ} \mathrm{C}$ for $2 \mathrm{~min}$ and finally extension at $72{ }^{\circ} \mathrm{C}$ for $10 \mathrm{~min}$ in a thermocycler. Amplified PCR products of 16 Sribosomal gene were separated on $1 \%$ agarose gel in $0.5 \times$ TE (Tris-EDTA) buffer containing 2 $\mu \mathrm{L}$ ethidium bromide (20 mg ml $\mathrm{m}^{-1}$ ) and sequencing of their 16S rRNA gene.

\section{Results and Discussion}

\subsection{Growth and colony morphology of isolates}

Total forty bacterial isolates took about $48 \mathrm{~h}$ to establish their growth on King's B agar. All the isolates developed small, medium, smooth and shiny colonies. About twenty isolates showed yellowish green, irregular, spreading, glistening, convex, opaque, viscid colonies. The remaining twenty isolates showed dull white irregular, spreading, glistening, convex, opaque, viscid colonies. Under microscopic studies, these isolates exhibited gram $^{-v e}$ nature with single, isolated, rod shaped cells with no endospores.

Among forty four Pseudomonas bacterial isolates, all the isolates showed positive results for gelatin hydrolysis, citrate utilization, oxidase test, catalase test. Nineteen isolates were positive for starch hydrolysis, thirty four isolates were positive for indole production, thirty isolates showed positive for methyl red test, thirty isolates were positive for Vogespraskauer test. Almost all isolates showed positive for $\mathrm{H}_{2} \mathrm{~S}$ production capability.

\subsection{Plant growth promoting properties}

Table 2 shows the Plant growth promoting properties of Pseudomonas isolates. Among forty four isolates, twenty eight isolates showed maximum PGP properties Among the twenty eight isolates, PS 21 showed highest P-solublization zone $(24.00 \pm 1.15 \mathrm{~mm})$, followed by PS-3 $(23.66 \pm 0.33 \mathrm{~mm})$, PS-1 $(18.66 \pm 0.88 \mathrm{~mm})$. Data on potassium solubilization activity show that out of twenty four isolates, PS 3 showed highest solubilization zone (22.67 mm), followed by, PS 14 (19.66 \pm 0.33 ), PS 35 (18.66 $\pm 0.33 \mathrm{~mm})$, PS $1(17.66 \pm 0.33 \mathrm{~mm})$. Out of twenty eight isolates IRP-7 showed highest Zinc solubilization zone $(27.00 \pm 1.73 \mathrm{~mm})$, followed by PS $15(25.66 \pm 0.33 \mathrm{~mm})$, PS

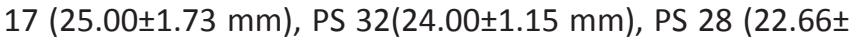
$0.33 \mathrm{~mm}$ ).The indole acetic acid production results revealed that, out of twenty eight isolates, PS 20 showed highest IAA production $\left(19.25 \pm 1.15 \mathrm{gg} \mathrm{ml}^{-1}\right)$, followed by PS $7(19.23 \pm 0.57$ $\left.\mu \mathrm{g} \mathrm{ml}^{-1}\right)$, PS $1\left(19.20 \pm 0.5 \mathrm{~g} \mathrm{ml}^{-1}\right)$, PS $14\left(18.70 \pm 0.14 \mu \mathrm{g} \mathrm{ml}^{-1}\right)$. Among twenty eight isolates, fourteen isolates (50\%) were 


\begin{tabular}{|c|c|c|c|c|c|c|c|c|c|}
\hline Isolates & $\begin{array}{l}\text { P solubi- } \\
\text { lization } \\
(\mathrm{mm})\end{array}$ & $\begin{array}{c}\text { K solubiliza- } \\
\text { tion }(\mathrm{mm})\end{array}$ & $\begin{array}{c}\mathrm{Zn} \\
\text { solublization } \\
(\mathrm{mm})\end{array}$ & $\begin{array}{c}\text { IAA produc- } \\
\text { tion } \\
\left(\mu \mathrm{g} \mathrm{m}^{-1}\right)\end{array}$ & $\begin{array}{c}\text { HCN } \\
\text { pro- } \\
\text { duction }\end{array}$ & $\begin{array}{l}\text { ACC } \\
\text { deami- } \\
\text { nase } \\
\text { activity }\end{array}$ & $\begin{array}{l}\text { Exo poly- } \\
\text { saccha- } \\
\text { rides pro- } \\
\text { duction }\end{array}$ & $\begin{array}{l}\text { Sidero- } \\
\text { phore } \\
\text { produc- } \\
\text { tion }\end{array}$ & $\begin{array}{l}\text { Antagonis- } \\
\text { tic activity } \\
\quad(\mathrm{mm})\end{array}$ \\
\hline PS 1 & $18.66 \pm 0.88$ & $17.66 \pm 0.33$ & $19.66 \pm 0.33$ & $19.20 \pm 0.5$ & ++ & +++ & +++ & ++ & $19.66 \pm 2.02$ \\
\hline PS 3 & $23.66 \pm 0.33$ & $22.66 \pm 0.88$ & $13.667 \pm 0.33$ & $14.00 \pm 1.15$ & ++ & - & & ++ & $23.66 \pm 0.33$ \\
\hline PS 4 & $14.00 \pm 1.52$ & $15.00 \pm 0.57$ & $19.00 \pm 0.57$ & $18.33 \pm 1.15$ & ++ & + & + & ++ & $25.00 \pm 0.57$ \\
\hline PS 5 & $10.33 \pm 0.88$ & $12.66 \pm 0.88$ & $14.00 \pm 1.15$ & $11.48 \pm 0.26$ & ++ & - & - & ++ & $24.33 \pm 0.33$ \\
\hline PS 7 & $17.33 \pm 0.33$ & $8.33 \pm 0.88$ & $27.00 \pm 1.73$ & $19.23 \pm 0.57$ & ++ & ++ & - & ++ & $19.33 \pm 0.33$ \\
\hline PS 8 & $12.00 \pm 1.15$ & $3.33 \pm 0.33$ & $3.33 \pm 0.33$ & $8.40 \pm 0.30$ & ++ & - & ++ & ++ & $12.66 \pm 0.88$ \\
\hline PS 10 & $7.00 \pm 1.00$ & $15.00 \pm 1.00$ & $15.00 \pm 1.15$ & $16.5 \pm 0.57$ & ++ & - & & ++ & $22.00 \pm 1.15$ \\
\hline PS 11 & $13.66 \pm 0.33$ & $16.00 \pm 1.155$ & $8.00 \pm 1.52$ & $6.50 \pm 0.28$ & ++ & +++ & ++ & ++ & $17.66 \pm 1.45$ \\
\hline PS 12 & $4.00 \pm 1.00$ & $1.00 \pm 0.00$ & $11.33 \pm 0.33$ & $15.97 \pm 2.50$ & ++ & + & - & ++ & $11.33 \pm 0.33$ \\
\hline PS 14 & $14.00 \pm 0.57$ & $19.66 \pm 0.33$ & $8.00 \pm 1.15$ & $18.70 \pm 0.14$ & ++ & & & ++ & $18.00 \pm 1.15$ \\
\hline PS 15 & $4.33 \pm 0.33$ & $7.33 \pm 0.33$ & $25.66 \pm 0.33$ & $4.23 \pm 0.00$ & ++ & +++ & +++ & ++ & $6.33 \pm 1.20$ \\
\hline PS 17 & $14.00 \pm 1.73$ & $6.00 \pm 0.57$ & $25.00 \pm 1.73$ & $5.21 \pm 1.15$ & ++ & - & - & ++ & $26.00 \pm 1.15$ \\
\hline PS 18 & $22.00 \pm 1.15$ & $15.00 \pm 1.732$ & $14.66 \pm 0.33$ & $18.20 \pm 1.15$ & ++ & + & - & ++ & $13.00 \pm 2.30$ \\
\hline PS 20 & $18.33 \pm 0.33$ & $4.00 \pm 1.00$ & $13.00 \pm 1.15$ & $19.25 \pm 1.15$ & ++ & - & - & ++ & $3.33 \pm 0.33$ \\
\hline PS 21 & $24.00 \pm 1.15$ & $12.33 \pm 0.33$ & $19.66 \pm 0.33$ & $15.57 \pm 0.21$ & ++ & + & + & ++ & $16.00 \pm 1.15$ \\
\hline PS-23 & $15.33 \pm 1.66$ & $13.00 \pm 1.15$ & $3.00 \pm 0.57$ & $12.23 \pm 0.57$ & ++ & ++ & + & ++ & $23.33 \pm 1.45$ \\
\hline PS 26 & $6.33 \pm 0.88$ & $3.00 \pm 0.57$ & $13.33 \pm 0.33$ & $5.36 \pm 0.57$ & ++ & - & - & ++ & $23.00 \pm 1.73$ \\
\hline PS 28 & $17.33 \pm 0.33$ & $12.66 \pm 0.33$ & $22.66 \pm 0.33$ & $1.50 \pm 0.25$ & ++ & - & - & ++ & $12.66 \pm 0.33$ \\
\hline PS 29 & $18.00 \pm 1.15$ & $3.00 \pm 0.57$ & $3.00 \pm 1.00$ & $9.25 \pm 1.155$ & ++ & + & - & ++ & $13.00 \pm 1.15$ \\
\hline PS 30 & $11.33 \pm 0.33$ & $9.33 \pm 0.33$ & $18.33 \pm 0.33$ & $14.52 \pm 1.62$ & ++ & +++ & +++ & ++ & $7.66 \pm 0.333$ \\
\hline PS 31 & $8.00 \pm 1.00$ & $13.00 \pm 1.15$ & $11.00 \pm 1.73$ & $13.00 \pm 2.51$ & ++ & - & - & ++ & $20.00 \pm 0.57$ \\
\hline PS 32 & $3.00 \pm 0.57$ & $13.66 \pm 0.33$ & $24.00 \pm 1.15$ & $8.32 \pm 1.73$ & ++ & - & - & ++ & $13.66 \pm 1.45$ \\
\hline PS 33 & $13.33 \pm 0.33$ & $12.00 \pm 1.15$ & $12.00 \pm 0.00$ & $15.21 \pm 0.48$ & ++ & - & - & ++ & $10.00 \pm 1.52$ \\
\hline PS 34 & $5.00 \pm 1.00$ & $7.00 \pm 1.00$ & $4.33 \pm 0.33$ & $18.30 \pm 1.00$ & ++ & ++ & ++ & ++ & $24.66 \pm 1.33$ \\
\hline PS 35 & $14.33 \pm 0.33$ & $18.66 \pm 0.33$ & $13.00 \pm 2.08$ & $12.26 \pm 0.57$ & ++ & - & - & ++ & $12.66 \pm 2.02$ \\
\hline PS-37 & $15.00 \pm 0.57$ & $17.33 \pm 0.33$ & $16.00 \pm 1.15$ & $15.547 \pm 0.22$ & ++ & + & ++ & ++ & $26.00 \pm 1.15$ \\
\hline PS 39 & $12.66 \pm 0.88$ & $6.00 \pm 1.732$ & $10.33 \pm 0.33$ & $10.89 \pm 4.78$ & ++ & - & - & ++ & $10.33 \pm 2.33$ \\
\hline PS 40 & $12.00 \pm 1.15$ & $4.33 \pm 0.33$ & $5.00 \pm 1.52$ & $13.63 \pm 0.18$ & ++ & ++ & + & ++ & $22.33 \pm 1.45$ \\
\hline SEm \pm & 0.924 & 0.829 & 0.928 & 1.373 & & & & & 1.285 \\
\hline $\begin{array}{l}C D \\
(p=0.05)\end{array}$ & 2.624 & 2.354 & 2.636 & 3.901 & & & & & 3.650 \\
\hline
\end{tabular}

positive for ACC deaminase production by utilization of ACC as the sole nitrogen source. Among fourteen isolates, four isolates showed strong (+++) ACCd production (PS 1, PS 11, PS 15, PS 30). Among the twenty eight isolates, eleven isolates (39 \%) positive for EPS production. Among eleven, three isolates showed strong (+++) for EPS production (PS 1, PS 15, PS-30). Similar results were reported by Fakedu (2013), that Pseudomonas fluorescens possessed promising properties which made it better biofertilizer bacteria. Twelve Pseudomonas fluorescenswere isolated from rhizospheric soil of faba bean and tested for phosphate solubilization. The results were in agreement with the findings of Norkina and Pumpynaskaya (1956) whoisolated two strains of Bacillus spp. and Pseudomonas from rhizosphere soils different crop plants as mineral potassium soluiblizers. Bapiri et al. (2012) evaluated zinc solubilizing ability of Pseudomonas fluorescens 
using zinc oxide, zinc carbonate and zinc sulphide in both plate and broth media assays. Zn solubilizing ability of 40 mentioned strains was studied with $\mathrm{ZnO}$ and $\mathrm{ZnCO}_{3}$ solutions in broth assay. The soluble zinc and $\mathrm{pH}$ were measured after five days. The results showed, that only 8 of 40 strains could form clearing zone in plate assay. Upadhyay and Srivastava (2010) reported that Pseudomonas fluorescens strain solubilized phosphorus and synthesized IAA. Renuga (2005) reported that plant growth promoting Pseudomonas pittida can utilize 1-aniinocyclopropanc- I-carboxylase as a sole nitrogen source because it possessed the unusual enzyme ACC dcaminase, which hydrolysis ACC to ammonia and a-ketobutyrate. Ali et al. (2013) isolated drought tolerant Pseudomonas isolates and these were screened for under both no stressed conditions as well as under minimum water potential $(-0.30 \mathrm{MPa})$. The strain Rdgp10 produced maximum amount of EPS $(3.22 \pm 0.04 \mathrm{mg} \mathrm{mg}$ protein $\left.^{-1}\right)$ under non-stressed condition, closely followed by isolate SorgP3 $\left(2.85 \pm 0.07 \mathrm{mg} \mathrm{mg} \mathrm{protein}^{-1}\right)$, sorgP4 $(2.75 \pm 0.06$ $\mathrm{mg} \mathrm{mg}$ protein $\left.{ }^{-1}\right)$, SunfP12 $\left(2.71 \pm 0.10 \mathrm{mg} \mathrm{mg} \mathrm{protein}^{-1}\right)$ and BriP15 (2.18 $\pm 0.25 \mathrm{mg} \mathrm{mg}$ protein $\left.{ }^{-1}\right)$.

All the isolates showed moderate amount $(++)$ of HCN production. Among twenty eight isolates, eight isolates showed strong (+++) siderophore production (PS 1, PS 3, PS 4, PS 11, PS 20, PS 35, PS 37, PS 40) and remaining twenty isolates showed moderate (++) siderophore production. Antifungal activity of 28 isolates was checked against Rhizoctonia solani and under in vitro conditions using PDA media. Based on inhibition zone out of 28 isolates, PS 17, PS 37 showed highest inhibition zone with $26.00 \pm 1.15 \mathrm{~mm}$, followed by PS $4(25.00 \pm 0.57 \mathrm{~mm})$, PS $34(24.66 \pm 1.33 \mathrm{~mm})$, PS $5(24.33 \pm 0.33 \mathrm{~mm})$, PS $3(23.66 \pm 0.33$ $\mathrm{mm}$ ). Siderophores such as pseudobacin and pyoverdin (yellow green fluorescent pigment of Pseudomonas bacteria) present high antimicrobial activity and affinity to ions of trivalent iron (Maksimov et al., 2011).

\subsection{Stress tolerance of Pseudomonas isolates}

Table 2 shows the abiotic stress tolerant selected Pseudomonas isolates. The results of different in vitro abiotic stress tolerance of Pseudomonas isolates, four isolates were showed growth at $\mathrm{pH}$ range from 4-10 (PS 1, PS 4, PS 26, PS 40), four isolates showed tolerance to $\mathrm{pH}$ range from 4-8 (PS 5,PS 7, PS 17, PS 20), three isolates showed tolerance to $\mathrm{pH}$ range from 4-7 (PS 15, PS 34, PS 39), three isolates showed tolerance to $\mathrm{pH}$ range from 6-10 (PS 11, PS 18, PS 32), two isolates showed tolerance to $\mathrm{pH}$ range from 6-8 (PS 14, PS 29), two isolates showed tolerance to $\mathrm{pH}$ range from 7-8 (PS 30, PS 31).

The results of salt tolerance ability reveals that, two isolates were showed tolerance to 1.5 to $20 \%$ of $\mathrm{NaCl}$ concentration (PS 4, PS 15), eight isolates were showed tolerance from 1.5 to $15 \%$ of $\mathrm{NaCl}$ concentration (PS 1, PS 11, PS 18, PS 26, PS 30 , PS 31, PS 32, PS 40), six isolates were showed tolerance from 1.5 to $10 \%$ of $\mathrm{NaCl}$ concentration (PS 5, PS 8, PS 14, PS 21, PS 23 , PS 29), three isolates were showed tolerance from 1.5 to $5 \%$ of $\mathrm{NaCl}$ concentration (PS 7, PS 17, PS 34, PS 37, PS 39).
The results of temperature tolerance ability of Pseudomonas isolates revealed that, three isolates showed tolerance to temperature from $20^{\circ} \mathrm{C}-50^{\circ} \mathrm{C}$ (PS 11, PS 15, PS 40), nine isolates were showed tolerance to temperature from $20{ }^{\circ} \mathrm{C}$ $45^{\circ} \mathrm{C}$ (PS 1 , PS 4 , PS 21, PS 23 , PS 28 , PS 30 , PS 32 , PS 34 , PS $37)$, one isolate was showed tolerance to temperature from $30{ }^{\circ} \mathrm{C}-50{ }^{\circ} \mathrm{C}$ (PS 7), two isolates were showed tolerance to temperature from $30^{\circ} \mathrm{C}-45^{\circ} \mathrm{C}$ (PS $12, \mathrm{PS} 18$ ).

The results of drought tolerance ability of Pseudomonas isolates revealed that, three isolates showed tolerance to water potential from - $0.05 \mathrm{Mpa}$ to- $0.73 \mathrm{Mpa}$ (PS 4, PS 15, PS 34), eight isolates were showed tolerance to water potential from - $0.05 \mathrm{Mpa}$ to $-0.30 \mathrm{Mpa}$ (PS 1,PS 7, PS 12, PS 18, PS 21, PS 23, PS 29, PS 37), seven isolates were showed tolerance to water potential at $-0.05 \mathrm{Mpa}$ (PS 10, PS 17, PS 20, PS 28, PS 31, PS 35, PS 39).

Similar results reported by Bhakthavatchal et al., 2013, Strain Pseudomonas aeruginosa FP6 was able to grow on up to 4.5 $\mathrm{M} \mathrm{NaCl}$, between 20 and $60{ }^{\circ} \mathrm{C}$ and at pH 5-10. Multifarious plant growth promoting activities of $P$. aeruginosa suggested its potential use in developing a cost-effective eco-friendly multifunctional biofertilizer.

Bacillus and Pseudomonas spp. with stress tolerance had proven ability to inhibit the growth of potential phytopathogenic fungi. Screening of bacterial strains for high temperature $\left(50^{\circ} \mathrm{C}\right)$, salinity $(7 \% \mathrm{NaCl})$, and drought $(-1.2$ $\mathrm{MPa}$ ) showed that stress tolerance was pronounced less in Pseudomonas isolates than in Bacillus strains. The reason behind this could be the formation of endospores by Bacillus isolates. Tolerance to drought was high in Pseudomonas strains than the other two stresses. Three strains, P8, P20 and P21 showed both salinity and temperature tolerance. P59 strain possessed promising antagonistic activity and drought tolerance (Praveen et al., 2014).

\subsection{Effect of heavy metals on Pseudomonas isolates}

The results of heavy metals tolerance of Pseudomonas revealed that, out of 28 isolates, fourteen isolates $(50 \%)$ showed growth on $50 \mathrm{\mu g} \mathrm{ml}^{-1}$ (PS $28\left(122 \times 10^{7} \mathrm{cfu} \mathrm{ml}^{-1}\right)>$ PS 7 $\left(120 \times 10^{7} \mathrm{cfu} \mathrm{m}^{-1}\right)$, PS $23\left(120 \times 10^{7} \mathrm{cfu} \mathrm{ml}^{-1}\right)$, PS $4\left(115 \times 10^{7} \mathrm{cfu}\right.$ $\left.\mathrm{ml}^{-1}\right)>$ PS $1\left(112 \times 10^{7} \mathrm{cfu} \mathrm{ml}^{-1}\right)$, IGP $8\left(112 \times 10^{7} \mathrm{cfu} \mathrm{ml}^{-1}\right)>$ PS 33 $\left(102 \times 10^{7} \mathrm{cfu} \mathrm{ml}^{-1}\right)$ and twelve isolates $(43 \%)$ showed growth on $100 \mathrm{mg} \mathrm{ml}^{-1}$ (PS $29\left(123 \times 10^{7} \mathrm{cfu} \mathrm{ml}^{-1}\right)>$ PS $28\left(50 \times 10^{7} \mathrm{cfu}\right.$ $\left.\mathrm{ml}^{-1}\right)$, PS $4\left(37 \times 10^{7} \mathrm{cfu} \mathrm{ml}^{-1}\right)$ on arsenic (As) enriched trypticase soy agar.

Fourteen isolates (50\%) showed cfu on $50 \mathrm{\mu g} \mathrm{ml}^{-1}$ (PS 32 $\left(125 \times 10^{7} \mathrm{cfu} \mathrm{ml}^{-1}\right)$, PS $5\left(112 \times 10^{7} \mathrm{cfu} \mathrm{ml}^{-1}\right)$, PS $20\left(112 \times 10^{7} \mathrm{cfu}\right.$ $\left.\mathrm{ml}^{-1}\right)$, PS $23\left(112 \times 10^{7} \mathrm{cfu} \mathrm{ml}^{-1}\right)$, PS $1\left(102 \times 10^{7} \mathrm{cfu} \mathrm{ml}^{-1}\right)$ and eleven isolates (43\%) were showed cfu on $100 \mathrm{\mu g} \mathrm{ml}^{-1}$ PS 28 $\left(86 \times 10^{7} \mathrm{cfu} \mathrm{ml}^{-1}\right)$, PS $20\left(63 \times 10^{7} \mathrm{cfu} \mathrm{ml}^{-1}\right)$ on cadmium (Cd) enriched trypticase soy agar respectively (Table 3 ).

Six isolates (21\%) and two isolates (7\%) showed cfu on 50 


\begin{tabular}{|c|c|c|c|c|}
\hline Isolates & $\mathrm{pH}$ range & $\mathrm{NaCl}$ concentration (\%) & Temperature $\left({ }^{\circ} \mathrm{C}\right)$ & Drought (Mpa) \\
\hline PS 1 & $4.0-10.0$ & $1.5-15$ & $20-45$ & -0.05 to -0.30 \\
\hline PS 3 & 7.0 & - & $20-30$ & - \\
\hline PS 4 & $4.0-10.0$ & $1.5-20$ & $20-45$ & -0.05 to -0.73 \\
\hline PS 5 & $4.0-8.0$ & $1.5-10$ & 30 & - \\
\hline PS 7 & $4.0-8.0$ & $1.5-5.0$ & $30-50$ & -0.05 to -0.30 \\
\hline PS 8 & 7.0 & $1.5-10$ & $20-30$ & - \\
\hline PS 10 & 7.0 & - & $20-30$ & -0.05 \\
\hline PS 11 & $6.0-10.0$ & $1.5-15$ & $20-50$ & \\
\hline PS 12 & 7.0 & - & $30-45$ & -0.05 to -0.30 \\
\hline PS 14 & $6.0-8.0$ & $1.5-10$ & $20-30$ & - \\
\hline PS 15 & $4.0-7.0$ & $1.5-20$ & $20-50$ & -0.05 to -0.73 \\
\hline PS 17 & $4.0-8.0$ & $1.5-5.0$ & 30 & -0.05 \\
\hline PS 18 & $6.0-10.0$ & $1.5-15$ & $30-45$ & -0.05 to -0.30 \\
\hline PS 20 & $4.0-8.0$ & - & $20-30$ & -0.05 \\
\hline PS 21 & 7.0 & $1.5-10$ & $20-45$ & -0.15 to -0.30 \\
\hline PS-23 & $6.0-7.0$ & $1.5-10$ & $20-45$ & -0.05 to -0.30 \\
\hline PS 26 & $4.0-10.0$ & $1.5-15$ & 30 & - \\
\hline PS 28 & 7.0 & - & $20-45$ & -0.05 \\
\hline PS 29 & $6.0-8.0$ & $1.5-10$ & 30 & $-0.05-0.30$ \\
\hline PS 30 & $7.0-8.0$ & $1.5-15$ & $20-45$ & - \\
\hline PS 31 & $7.0-8.0$ & $1.5-15$ & 30 & -0.05 \\
\hline PS 32 & $6.0-10.0$ & $1.5-15$ & $20-45$ & - \\
\hline PS 33 & 7.0 & - & $20-30$ & - \\
\hline PS 34 & $4.0-7.0$ & $1.5-5.0$ & $20-45$ & -0.05 to -0.73 \\
\hline PS 35 & $7.0-8.0$ & - & 30 & -0.05 \\
\hline PS-37 & 7.0 & $1.5-5.0$ & $20-45$ & $-0.15-0.30$ \\
\hline PS 39 & $4.0-7.0$ & $1.5-5.0$ & 30 & -0.05 \\
\hline PS 40 & $4.0-10.0$ & $1.5-15$ & $20-50$ & - \\
\hline
\end{tabular}

$\mu \mathrm{g} \mathrm{ml} l^{-1}$ (PS $\left.558 \times 10^{7} \mathrm{cfu} \mathrm{ml}^{-1}\right)$, PS-14 $\left(46 \times 10^{7} \mathrm{cfu} \mathrm{ml}^{-1}\right)$, PS 20 $\left(42 \times 10^{7} \mathrm{cfu} \mathrm{ml}^{-1}\right)$, PS $37\left(42 \times 10^{7} \mathrm{cfu} \mathrm{ml}^{-1}\right)$ and $100 \mathrm{\mu g} \mathrm{ml}^{-1} \mathrm{PS}-$ $37\left(32 \times 10^{7} \mathrm{cfu} \mathrm{ml}^{-1}\right)$ PS $32\left(30 \times 10^{7} \mathrm{cfu} \mathrm{m}^{-1}\right)$ on mercury $(\mathrm{Hg})$ enriched trypticase soy agar respectively.

Twelve isolates (43\%) and seven isolates (25\%) showed cfu on $50 \mathrm{mg} \mathrm{ml}^{-1}$ (PS $32\left(120 \times 10^{7} \mathrm{cfu} \mathrm{ml}^{-1}\right)$, PS $20\left(113 \times 10^{7} \mathrm{cfu}\right.$ $\left.10^{7} \mathrm{ml}^{-1}\right)$, PS $18\left(103 \times 10^{7} \mathrm{cfu} \mathrm{ml}^{-1}\right)$, PS $3\left(80 \times 10^{7} \mathrm{cfu} \mathrm{m}^{-1}\right)$, PS 5 $\left(72 \times 10^{7} \mathrm{cfu} \mathrm{ml}^{-1}\right)$ and $100 \mathrm{~g} \mathrm{ml}^{-1}$ (PS $18\left(43 \times 10^{7} \mathrm{cfu} \mathrm{ml}^{-1}\right)$, PS 32 $\left(43 \times 10^{7} \mathrm{cfu} \mathrm{ml}^{-1}\right)$, PS $3\left(33 \times 10^{7} \mathrm{cfu} \mathrm{ml}^{-1}\right)$, PS $\left.5\left(33 \times 10^{7} \mathrm{cfu} \mathrm{ml}^{-1}\right)\right)$ on manganese $(\mathrm{Mn})$ enriched trypticase soy agar respectively. Similar results reported by Kowalczyk et al. (2016) tested ability to remove $\mathrm{Hg}$ from the liquid medium and the effect of the various $\mathrm{pH}$ and mercury concentrations in the environment on bacterial strains growth kinetics. The selected strains were identified by analysis of the $16 \mathrm{~S}$ ribosome subunit coding sequences as Pseudomonas syringae. It was demonstrated that $P$. syringae was able to remove almost entire metal from medium after 120 hours of incubation. Huixu et al. (2012) isolated a mercury resistant strain 2 from waste treatment plant. It was identified as Pseudomonas putida and designated as Pseudomonas putidaon the basis of morphological and biochemical analysis in combination with phylogenetic analysis. Strain could tolerate and aerobically grow in the medium containing up to $50 \mathrm{mg} \mathrm{dm}{ }^{3} \mathrm{Hg}$ (II). Raja et al. (2006) stated that growth rate of the sewage isolates in the presence of heavy metal $(\mathrm{Cd}, \mathrm{Ni}, \mathrm{As}$ and $\mathrm{Pb})$ were consistently slower than that of the control, In this study, Pseudomonas sps were resistance to cadmium $7 \mathrm{mM}$ in TY agar plate (Table 4). 


\begin{tabular}{|c|c|c|c|c|c|c|c|c|}
\hline \multirow[t]{3}{*}{ Isolates } & \multicolumn{8}{|c|}{ Heavy metal tolerance $\left(\times 10^{7} \mathrm{cfu} \mathrm{\textrm {ml } ^ { - 1 } )}\right.$} \\
\hline & \multicolumn{2}{|c|}{ As } & \multicolumn{2}{|c|}{$\mathrm{Cd}$} & \multicolumn{2}{|c|}{$\mathrm{Hg}$} & \multicolumn{2}{|c|}{$\mathrm{Mn}$} \\
\hline & $50 \mu \mathrm{g} \mathrm{ml}^{-1}$ & $100 \mu \mathrm{g} \mathrm{ml}^{-1}$ & $50 \mu \mathrm{g} \mathrm{ml}^{-1}$ & $100 \mu \mathrm{g} \mathrm{ml}^{-1}$ & $50 \mu \mathrm{g} \mathrm{ml}^{-1}$ & $100 \mu \mathrm{g} \mathrm{ml}^{-1}$ & $50 \mu \mathrm{g} \mathrm{ml}^{-1}$ & $100 \mu \mathrm{g} \mathrm{ml}^{-1}$ \\
\hline PS 1 & 112 & 32 & 102 & 50 & - & - & 70 & 30 \\
\hline PS 3 & - & - & - & - & - & - & 80 & 33 \\
\hline PS 4 & 115 & 37 & 53 & - & - & - & - & - \\
\hline PS 5 & 79 & 31 & 112 & 50 & 58 & - & 72 & 33 \\
\hline PS 7 & 120 & 30 & - & - & - & - & - & - \\
\hline PS 8 & 112 & 30 & - & - & - & - & - & - \\
\hline PS 10 & - & - & - & - & - & - & - & - \\
\hline PS 11 & - & - & 100 & 35 & - & - & 65 & 30 \\
\hline PS 12 & - & - & - & - & - & - & - & - \\
\hline PS 14 & 86 & 31 & 70 & 30 & 46 & - & 30 & - \\
\hline PS 15 & 112 & 32 & 56 & - & - & - & - & - \\
\hline PS 17 & - & - & - & - & - & - & - & - \\
\hline PS 18 & 58 & 32 & - & - & - & - & 103 & 43 \\
\hline PS 20 & 42 & - & 112 & 63 & 42 & - & 113 & 30 \\
\hline PS 21 & - & - & - & - & - & - & - & - \\
\hline PS-23 & 120 & 32 & 112 & 50 & - & - & 52 & - \\
\hline PS 26 & - & - & 100 & 35 & - & - & 65 & - \\
\hline PS 28 & 122 & 50 & 85 & 86 & - & - & 58 & - \\
\hline PS 29 & 98 & 123 & 43 & 50 & - & - & 56 & - \\
\hline PS 30 & - & - & - & - & - & - & - & - \\
\hline PS 31 & - & - & - & - & 30 & - & - & - \\
\hline PS 32 & 36 & - & 125 & 50 & 20 & 30 & 120 & 43 \\
\hline PS 33 & 102 & 32 & 78 & 32 & - & - & - & - \\
\hline PS 34 & - & - & & & 58 & - & - & - \\
\hline PS 35 & - & - & - & - & - & - & - & - \\
\hline PS-37 & - & - & - & - & 42 & 32 & - & - \\
\hline PS 39 & - & - & - & - & - & - & - & - \\
\hline PS 40 & - & - & - & - & - & - & - & - \\
\hline
\end{tabular}

For PGP properties, among Pseudomonas isolates, PS 1 showed efficient P-solubilization, K- solubilization, Zn solubilization, and biocontrol activity. PS 15 showed efficient Zn-solubilization, and other PGP properties. IRP-7 showed good P-solubilization, Zn-solubilization, IAA production and other PGP properties. For plant growth promoting properties and abiotic stress, PS 15 was found efficient in $\mathrm{Zn}$ - solubilization with considerable other PGP properties. It was tolerant to $\mathrm{pH}$ stress $(4,6)$, temperature stress $\left(40{ }^{\circ} \mathrm{C}, 45^{\circ} \mathrm{C}, 50^{\circ} \mathrm{C}\right)$, salt stress $(5 \%, 10 \%)$, drought stress (-0.15 $\mathrm{MPa},-0.30 \mathrm{MPa},-0.73 \mathrm{Mpa})$, and heavy metals As and $\mathrm{Cd}$ only. PS 1 showed good P-solubilization, $\mathrm{K}$ - solubilization, $\mathrm{Zn}$ solubilization withbiocontrol activity. It tolerated $\mathrm{pH}$ stress $(4,6,8,10,12)$, temperature stress $\left(40^{\circ} \mathrm{C}\right.$, $\left.45^{\circ} \mathrm{C}\right)$, salt stress $(5 \%, 10 \%, 15 \%)$, drought stress (-0.15 MPa, $-0.30 \mathrm{MPa}$ ) and tolerant to heavy metal toxicity $\mathrm{As}, \mathrm{Cd}$ and $\mathrm{Mn}$ Identification of bacterial strains based on 16S rRNA gene sequence is given in Table In the present study the sequenced PCR products of effective bacterial isolates were matched with the available sequences in the GenBank database. BLAST Search results through NCBI showed $99 \%$ similarity of PS 15 with Pseudomonas fluorescens, 99\% similarity of PS 1 with Pseudomonas fluorescens.

\section{Conclusion}

In regard to isolates having PGP properties and biocontrol 
activity, PS-3 can be considered efficient isolate. However, none of the isolates showed commonly PGP properties, biocontrol activity and tolerance to abiotic stress conditions. A combination of isolates PS-3 and PS-1 could be a good combination for the purpose. Effective Pseudomonas isolates were identified as Pseudomonas fluorescens. The plant growth promoting Pseudomonas isolates identified from the research work presented could be screened further under in vivo conditions of abiotic stress conditions from different soils with different crops for confirming their use as bioinoculants.

\section{Acknowledgement}

Author acknowledge the financial support extended by the University Grants Commission for National fellowship year 2016-17.

\section{References}

Alami, Y., Achouak, W., Marol, C., Heulin, T., 2000. Rhizosphere soil aggregation and plant growth promotion of sunflowers by an exo polysaccharideproducing Rhizobium sp. strain isolated from sunflower roots. Applied and Environmental Microbiology 66, 3393-3398.

Ali, S.Z., Sandhya, V, Rao, L.V., 2013. Isolation and characterization of drought tolerant ACC deaminase and exo polysaccharide producing fluorescent Pseudomonas sp. Annals of Microbiology 5, 1-10.

Bapiri, A., Asgharzadeh, A., Mujallali, H., Khavazi, Pazira, E., 2012. Evaluation of Zinc solubilization potential by different strains of fluorescent pseudomonads. Journal of Applied Sciences and Environmental Management 16, 295-298.

Bhakthavatchalu, S., Shivakumar, S., Shankar, B.S., 2013. Characterization of multiple plant growth promotion traits of Pseudomonas aeruginosa FP6, a potential stress tolerant biocontrol agent. Annals of Biological Research 4(2), 214-223.

Castric, K.F., Castric, P.A., 1983. Method for rapid detection of cyanogenic bacteria. Applied and Environmental Microbiology 45, 700-702.

Dimkpa, C., Svatos, A., Merten, D., Buchel, G., Kothe, E., 2008. Hydroxamate siderophores produced by Streptomyces acidiscabies E13 bind nickel and promote growth in cowpea (VignaunguiculataL.) under nickel stress. Canadian Journal of Microbiology 54, 163-172 .

Djedidi, S., Yokoyama, T., Ohkama-Ohtsu, N., Risal, C.P., Abdelly, C., Sekimoto, H., 2011. Stress tolerance andsymbiotic and phylogenic features of root nodulebacteria associated with medicagospecies in differentbioclimatic regions of tunisia. Microbes and Environments 26, 36-45.

Dubey, R.C., Maheshwari, D.K., Aeron, A., Kumar, B., Kumar, S., 2012. Integrated approach for disease management and growth enhancement of Sesamum indicum L. utilizing Azotobacter chroococcum TRA2 and chemical fertilizer. World Journal of Microbiology and Biotechnology 28, 3015 Hydroxamate siderophores, 3024.

Fakedu, A., 2013. Isolation of Pseudomonas fluorescens from rhizospheric soil of faba bean and assessment of their Phosphate solubility: in vitro study, Ethiopia. Scholars Academic Journal of Biosciences 1(7), 346-351.

Farooq, M., Wahid, A., Kobayashi, N., Fujita, D., Basra, S.M.A., 2009. Plant drought stress: effects, mechanisms and management. Agronomy for Sustainable Development 29 (1), 185-212.

Gray, E.J., Smith, D.L., 2005. Intracellular and extracellularPGPR: commonalities and distinctions in the plant-bacterium signaling processes. Soil Biology \& Biochemistry 37395-37412.

Grover, M., Ali, Sk. Z., Sandhya, V., Rasul, A., Venkateswarlu, B., 2011. Role of microorganisms in adaptation of agriculture crops to abiotic stresses. World Journal of Microbiology and Biotechnology 27, 1231-1240 .

Holt, J.G., Kreig, N.R., Sneath, P.H.A., Staley, J.T., Williams, S.T., 1984. Bergy's manual of systematic bacteriology. Ninth edition, 151-168.

Hui, X.U., De-jucao., Zhong-fengtian., 2012. Isolation and identification of a mercury resistant strain. Environment Protection Engineering 38(4), 67-75.

Kowalczyk, A., OzgeEyice., Schafer, H., Price, O.R., 2015. Characterization of $p$-Nitro phenol degrading bacterial communities in river water by using functional markers and stable isotope probing. Applied and Environmental Microbiology 8(1), 19, 6890-6900.

Landa, B.B., Navas-Cortes, J.A., Jimenez-Diaz, R.M., 2004. Influence of temperature on plant-rhizobacteria interactions related to biocontrol potential for suppression of fusarium wilt of chickpea. Plant Pathology 53(3), 341-352.

Maksimov, I.V., Abizgildina, R.R., Pusenkova, L.I., 2011. Plant growth promoting microorganisms as alternative to chemical protection from pathogens. Prikl Biokhim Microbiology 47 (4), 373-385.

Michel, B.E., Kaufmann, M.R., 1973. The osmotic potential of polyethylene glycol 6000. Plant Physiology, 51, 914916.

Norkina, S.P., Pumpyansakya, L.V., 1956. Certain properties of silicate bacteria dokl. Crop Science Society of Japan 28, 35-40.

Pikovskaya, R.I., 1948. Mobilization of phosphorus in soil connection with the vital activity of some microbial species.Microbiologia 17, 362-370.

Prajapati, M.C., Modi, H.A., 2012. Isolation of two potassium solubilizing fungi from ceramic industry soils. Life sciences Leaflets 5, 71-75.

Praveen, G.K., Mir Hassan Ahmed, S.K., Desai, S., Leo, E.D.A., Abdul, R., 2004. In vitro Screening for Abiotic Stress Tolerance in Potent Bio control and Plant Growth 
Promoting Strains of Pseudomonas and Bacillus spp. International journal of bacteriology, 1-6.

Raja, P., Una, S., Gopal, H., Govindarajan, K., 2006. Impact of bio inoculants consortium on rice root exudates, biological nitrogen fixation and plant growth. Journal Biological Sciences 6, 815-823.

Renuga, G., 2005. Characterization of ACC Deaminase in plant growth promoting Pseudomonas from tannery sludge. Journal of Industrial Pollution Control 21(2), 361-370.

Sandhya, V., Ali, S.Z., Grover, M., Reddy, G., Bandi, V., 2009. Drought-tolerant plant growth promoting Bacillus spp. effect on growth, osmolytes and antioxidant status of maize under drought stress.Journal of Plant Interactions 6, 1-14.

Saravanakumar, D., Samiyappan R., 2007. ACC deaminase from Pseudomonas fluorescensmediated saline resistance in groundnut(Arachis hypogaea) plants. Journal of Applied Microbiology 102, 1283-1292.

Saravanan, V.S., Subramoniam, S.R., Raj, S.A.,2003. Assessing in vitro solubilization potential of different zinc solubilizing bacterial isolates. Brazilian Journal of Microbiology 34, 121-125.

Schwyn, B., Neilands, J.B., 2003. Universal chemical assay for the detection and determination of siderophores. Analytical Biochemistry 160, 47-56.

Skidmore, A.M., Dickinson, C.H., 1976. Colony interaction and hyphal interference between Septorianodorum and phylloplane fungi. Transactions and Journal of the British Ceramic Society 66, 57-74.
Tokala, R.K., Strap, J.L., Jung, C.M., Crawford, D.L., Salove, H., Deobald, L.A., Bailey, F.J., Morra, M.J., 2002. Novel plant-microbe rhizosphere interaction involving $S$. lydicusWYEC108and the pea plant (Pisum sativum). Applied and Environmental Microbiology 68, 2161-2171.

Upadhyay, A., Srivastava, S., 2002. Evaluation of multiple plant growth promoting traits of an isolate of Pseudomonas fluorescens strain Psd.: Indian Journal of Experimental Biology 48, 601-609.

Venkateswarlu, B., Desai, S., Prasad, Y.G., 2008. Agriculturally important microorganisms for stressed ecosystems: Challenges in technology development and application". In: Khachatourians GG, Arora DK, Rajendran TP, Srivastava AK (eds) Agriculturally important Microorganisms, Academic World, Bhopal, (1), 225-246.

Vlassak, K.L., Van, H., Duchateau, L., 1992. Isolation and characterization of fluorescent Pseudomonas associated with the roots of rice and banana grown in Srilanka. Plant and soil 145, 51-63.

Wegulo, S., 2012. Factors Influencing Deoxynivalenol Accumulation in Small Grain Cereals.Toxins (Basel) 4(11), 1157-1180.

Weller, D.M., Landa, B.B., Mavrodi, O.V., Schroeder, K.L., De LaFuente, L., Bankhead, S.B., Molar, R.A., Bonsall, R.F., Mavrodi, D.V., Thomashow, L.S., 2007. Role of 2,4-diacetylphloroglucinol-producing fluorescent Pseudomonas spp. in the defense of plant roots. Plant Biology 9, 4-20. 